\title{
On a Pathology in Indefinite Metric Inner Product Space
}

\author{
Huzihiro Araki \\ Research Institute for Mathematical Sciences, Kyoto University, Kyoto 606, Japan
}

\begin{abstract}
A pathology related to an indefinite metric, which has been pointed out by Ito in connection with construction of a two dimensional quantum field model at a finite cutoff, is mathematically analyzed in a simple model. It is found for a model Hamiltonian with a parameter in an indefinite metric inner product space that eigenvalues with a complete set of eigenvectors changes suddenly from positive integers to negative integers as a parameter crosses a critical value (the Hamiltonian being skew selfadjoint with absolutely continuous spectrum on a pure imaginary axis at the critical value of the parameter), if a fixed (positive definite Hilbert space) topology is used in the completion of the underlying indefinite metric inner product space. However it is also found that if the topology is varied with the parameter of the Hamiltonian in the manner similar to analytic continuation, then the Hamiltonian keeps positive integer eigenvalues with a complete set of eigenvectors.
\end{abstract}

\section{Introduction}

It is a great pleasure to dedicate this article to the $60^{\text {th }}$ birthday of Professor Rudolf Haag. In one of his pioneering works on algebraic approach in quantum field theory, Haag gave examples of (bilinear) Hamiltonians whose vacuum vectors give rise to inequivalent representations of canonical commutation relations [1]. In this article, we use similar examples on an indefinite metric Hilbert space to show some pathological phenomena in such a space and a possible method of overcoming such a pathology.

Ito [2] tried to apply the original method of constructive field theory to study the limit of cut-off quantum electrodynamics in one space and one time dimension. The model is exactly solvable in principle by Bogoliubov transformations. Ito works on a Fock space with an indefinite metric and studies what can be considered the vacuum vector for the cut-off Hamiltonian, by applying an appropriate Bogoliubov transformation (mathematically unbounded operators) on the free vacuum vector. It turns out that there is a finite value of the cut-off 
parameter beyond which the Bogoliubov transformation can not be defined as a closable operator whose domain contains the free vacuum vector, and thus presents a mathematical barrier in obtaining the vacuum vector for Hamiltonians with the cut-off parameter larger than a certain finite value.

The main aim of this article is to clarify the exact mathematical nature of the above mentioned difficulty and to suggest a method to circumvent it, in the case of a simplified model. We show that the Hamiltonian $H_{\theta}$ in our model has positive integer eigenvalues with a complete set of eigenvectors for values of the parameter $\theta$ below a critical value, negative integer eigenvalues with a complete set of eigenvectors for values of the same parameter above the critical value, and is skew self-adjoint with absolutely continuous spectrum on the pure imaginary axis at the critical value of the parameter. The critical value of the parameter exactly corresponds to a point where the Bogoliubov transformation diagonalizing the Hamiltonian can no longer be defined as a closable operator whose domain contains the free vacuum vector.

The situation just described sounds like denying the possibility of construction of the model by taking the limit of infinite cut-off because a difficulty may arise already at a finite cut-off. Actually there is a subtle point related to the idefinite metric. Originally the one-parameter family of Hamiltonians in our model is defined on the set $D_{0}$ of all finite linear combinations of vectors for a finite number of particles in an indefinite metric inner product space. In order to be able to do an analysis, we introduce a (positive definite) Hilbert space topology $\tau_{0}$ in $D_{0}$ and consider its completion as a Hilbert space. The above mentioned difficulty occurs in this completed space.

The indefinite metric on $D_{0}$ is supposed to be relevant to physical interpretation of the model but the positive definite metric and the associated topology $\tau_{0}$ which we used in defining the completion of $D_{0}$ is not intrinsic to the problem. In fact we can introduce a one parameter family of (positive definite) Hilbert space metrics $\tau_{\theta}$ on $D_{0}$ such that $H_{\theta^{\prime}}$ has positive integer eigenvalues with a complete set of eigenvectors in the $\tau_{\theta}$-completion of $D_{0}$ if $\theta^{\prime}-\theta$ is small and, in particular, $H_{\theta}$ is selfadjoint and positive there. In fact, all $H_{\theta^{\prime}}$ in our model are mutually related by a Bogoliubov transformation, which can be implemented by a well-defined closable operator (preserving the indefinite metric) on the $\tau_{\theta}$-completion of $D_{0}$ if $\left|\theta-\theta^{\prime}\right|$ is below a critical value.

This result shows the importance of the choice of the topology for the indefinite metric inner product space on which we want to define a Hamiltonian: For one choice of topology, for example $\tau_{0}$, we can deal with a Hamiltonian $H_{\theta}$ for a certain range of the parameter $\theta$, but in order to increase the value of the parameter $\theta$ while retaining some positive character of the spectrum of $H_{\theta}$, we have to use another topology, say $\tau_{\theta_{1}}$. The new topology allows us to deal with $H_{\theta}$ for the value of $\theta$ in the neighbourhood of $\theta_{1}$. We may continue this way, like an analytic continuation, and keep the positive point spectrum of $H_{\theta}$. In fact, for each $\theta$, there is a topology $\tau_{\theta}$ which makes $H_{\theta}$ positive selfadjoint in the $\tau_{\theta}$-completion of the space. Actually, if we insist on the initial domain $D_{0}$, we can not introduce the topology $\tau_{\theta}$ for large values of $\theta$. However we introduce another domain $D_{\theta}$ in the $\tau_{\theta}$-completion of $D_{0}$ such that $\tau_{\theta^{\prime}}$ are well-defined on $D_{\theta}$ if $\left|\theta^{\prime}-\theta\right|$ is not too large. In this manner we can continue the above process to an arbitrarily large $\theta$ and find 
the same spectrum of $H_{\theta}$ as one would by a naive diagonalization by Bogolubov transformations.

The model used here is a special example and it is not at all clear whether the same procedure is applicable in a general situation. However it does suggest a new viewpoint, which might be overlooked otherwise, and it might be useful in some case as a heuristic method of finding a model with desirable properties in the limit of no cut-off.

\section{Main Results}

The complex vector space $D_{0}$ is the set of all finite linear combinations of linearly independent vectors $\Psi(n, m ; 0)$ indexed by $n=0,1,2, \ldots$ and $m=0,1,2, \ldots$ An indefinite inner product is defined on $D_{0}$ such that

$$
\left\langle\Psi_{0}(n, m ; 0), \Psi_{0}\left(n^{\prime}, m^{\prime} ; 0\right)\right\rangle= \begin{cases}n ! m !(-1)^{m} & \text { if } n=n^{\prime}, m=m^{\prime} \\ 0 & \text { otherwise }\end{cases}
$$

Annihilation and creation operators are defined as follows:

$$
\left.\begin{array}{rl}
a^{*} \Psi_{0}(n, m ; 0) & =\Psi_{0}(n+1, m ; 0), \\
b^{*} \Psi_{0}(n, m ; 0) & =\Psi_{0}(n, m+1 ; 0), \\
a \Psi_{0}(n, m ; 0) & =n \Psi_{0}(n-1, m ; 0) \quad(=0 \quad \text { if } \quad n=0), \\
b \Psi_{0}(n, m ; 0) & =-m \Psi_{0}(n, m-1 ; 0)(=0 \quad \text { if } \quad m=0) .
\end{array}\right\}
$$

They satisfy $\left[a, a^{*}\right]=\left[b^{*}, b\right]=1, \quad a \Omega_{0}=b \Omega_{0}=0 \quad$ for $\quad \Omega_{0} \equiv \Psi_{0}(0,0 ; 0)$ and $\Psi(n, m ; 0)=\left(a^{*}\right)^{n}\left(b^{*}\right)^{m} \Omega_{0}$. Relative to the indefinite metric, $a^{*}$ and $a$ are adjoint of each other on $D_{0}$ and the same holds for $b^{*}$ and $b$.

We define

$$
H_{\theta}=\left(a^{*} a-b b^{*}\right) \cos 2 \theta+\left(a^{*} b^{*}+a b\right) \sin 2 \theta .
$$

If we write

$$
\left.\begin{array}{l}
a_{\theta}=\cos \theta a+\sin \theta b^{*} \\
b_{\theta}=\cos \theta b-\sin \theta a^{*}
\end{array}\right\}
$$

then, denoting the adjoint on $D_{0}$ by $*$, we have

$$
H_{\theta}=a_{\theta}^{*} a_{\theta}-b_{\theta} b_{\theta}^{*} .
$$

A positive definite inner product $(,)_{0}$ on $D_{0}$ is defined by

$$
\begin{gathered}
\left(\Phi_{1}, \Phi_{2}\right)_{0}=\left\langle\Phi_{1}, \eta_{0} \Phi_{2}\right\rangle, \\
\eta_{0} \Psi_{0}(n, m ; 0)=(-1)^{m} \Psi_{0}(n, m ; 0) .
\end{gathered}
$$

The completion of $D_{0}$ with respect to the topology $\tau_{0}$ given by this inner product will be denoted $\mathscr{H}_{0}$. The closure of $H_{\theta}$ in $\mathscr{H}_{0}$ is denoted by $\bar{H}_{\theta}^{0}$.

Theorem 1. (1) If $|\theta|<\pi / 4$, then there exists a complete set of vectors $\Psi_{0}(n, m ; \theta)$ in $\mathscr{H}_{0}$ such that

$$
\bar{H}_{\theta}^{0} \Psi_{0}(n, m ; \theta)=(n+m+1) \Psi_{0}(n, m ; \theta) .
$$


(2) If $|\theta|=\pi / 4, \bar{H}_{\theta}^{0}$ is skew self-adjoint with absolutely continuous spectrum on the pure imaginary axis.

(3) If $\pi / 4<\theta<3 \pi / 4$, then there exists a complete set of vectors $\Psi_{0}(n, m ; \theta)$ in $\mathscr{H}_{0}$ such that

$$
\bar{H}_{\theta}^{0} \Psi_{0}(n, m ; \theta)=-(n+m+1) \Psi_{0}(n, m ; \theta) .
$$

Remark 1. Since $H_{\theta+\pi}=H_{\theta}$, Theorem 1 covers all real values of $\theta$. Since $H_{\theta+(\pi / 2)}=-H_{\theta}$, Theorem 1 (3) follows from Theorem 1 (1).

Remark 2. Let $D_{0}(k)$ be the set of all finite linear combinations of $\Psi_{0}(n, m ; 0)$ with $n-m=k$ and let $\mathscr{H}_{0}(k)$ be its closure in $\mathscr{H}_{0}$. Then $D_{0}$ is the direct sum of $D_{0}(k)$ for all integer values of $k, \eta D_{0}(k)=D_{0}(k)$, and hence $\mathscr{H}_{0}=\sum_{k=-\infty}^{\infty} \oplus \mathscr{H}_{0}(k)$. Furthermore, each $\mathscr{H}_{0}(k)$ is invariant under $\bar{H}_{\theta}^{0}, \Psi_{0}(n, m ; \theta) \in \mathscr{H}_{0}(k)$ if and only if $n-m=k$ (as is shown in Sect. 3) and the restriction of $\bar{H}_{\theta}^{0}$ to each $\mathscr{H}_{0}(k)$ has simple eigenvalues at $|k|+2 n+1, n=0,1,2, \ldots$, with a complete set of eigenvectors.

Remark 3. Let $A^{\dagger 0}$ denote the adjoint of $A$ relative to the positive definite inner product (2.6). Then $a^{\dagger 0} \supset a^{*}$ and $b^{\dagger 0} \supset-b^{*}$. Therefore $\left(a \pm i b^{*}\right)^{\dagger 0}\left(a \pm i b^{*}\right) \geqq 0$ implies

$$
\left(a^{*} a-b b^{*}\right) \geqq \pm i\left(a^{*} b^{*}+a b\right) \geqq-\left(a^{*} a-b b^{*}\right)
$$

as a sesquilinear form on $\left(D_{0},(,)_{0}\right)$. Hence $\bar{H}_{\theta}^{0}$ is sectorial $(|\theta|<\pi / 4)$. Since the residual spectrum of $\bar{H}_{\theta}^{0}$, if any, is limited to positive integers due to Theorem 1 (1), $\bar{H}_{\theta}^{0}$ is $m$-sectorial. (Alternatively, we can obtain the same conclusion from a general theorem that a sectorial operator with a dense set of analytic vectors $\left(D_{0}\right.$ for $\left.\bar{H}_{\theta}^{0}\right)$ is $m$-sectorial.) Since $\operatorname{Re} \bar{H}_{\theta}^{0}=\bar{H}_{0}^{0}$ has a compact resolvent, the same holds for $\bar{H}_{\theta}^{0}$. (Theorem VI-3.3 [3].) By Theorem 1 (1), the spectrum of $\bar{H}_{\theta}^{0}$ is positive integers for $|\theta|<\pi / 4$. By Remark 1, it is negative integers for $\pi / 4<\theta<3 \pi / 4$.

We now consider a one-parameter family of positive definite inner product on $D_{0}$ uniquely determined by the following:

$$
\left(\Psi_{0}(n, m ; 0), \Psi_{0}\left(n^{\prime}, m^{\prime} ; 0\right)\right)_{\theta}=\left(\Psi_{0}(n, m ;-\theta), \Psi_{0}\left(n^{\prime}, m^{\prime} ;-\theta\right)\right)_{0},
$$

where $|\theta|<\pi / 4$. Since the indefinite metric (2.1) can obviously be extended to a non-degenerate indefinite inner product on $\mathscr{H}_{0}$ and the vectors $\Psi_{0}(n, m ;-\theta)$ for a fixed $\theta$ are mutually orthogonal and not isotropic relative to the indefinite metric (as is shown in Sect. 3), $\Psi_{0}(n, m ;-\theta)$ are linearly independent and hence (2.11) is positive definite on $D_{0}$. Let $\mathscr{H}_{\theta}$ be the completion of $D_{0}$ with respect to the topology $\tau_{\theta}$ given by the inner product (2.11) and $\bar{H}_{\theta^{\prime}}^{\theta}$ be the closure of $H_{\theta^{\prime}}$ in $\mathscr{H}_{\theta}$.

Theorem 2. (1) If $\left|\theta-\theta^{\prime}\right|<\pi / 4$, then there exists a complete set of vectors $\Psi_{\theta}\left(n, m ; \theta^{\prime}\right)$ in $\mathscr{H}_{\theta}$ such that

$$
\bar{H}_{\theta^{\prime}}^{\theta} \Psi_{\theta}\left(n, m ; \theta^{\prime}\right)=(n+m+1) \Psi_{\theta}\left(n, m ; \theta^{\prime}\right) .
$$

(2) If $\left|\theta-\theta^{\prime}\right|=\pi / 4, \bar{H}_{\theta^{\prime}}^{\theta}$ is skew self-adjoint with absolutely continuous spectrum on the pure imaginary axis. 
(3) If $\pi / 4<\theta^{\prime}-\theta<3 \pi / 4$, then there exists a complete set of vectors $\Psi_{\theta}\left(n, m ; \theta^{\prime}\right)$ in $\mathscr{H}_{\theta}$ such that

$$
\bar{H}_{\theta^{\prime}}^{\theta} \Psi_{\theta}\left(n, m ; \theta^{\prime}\right)=-(n+m+1) \Psi_{\theta}\left(n, m ; \theta^{\prime}\right) .
$$

Remark 4. $\bar{H}_{\theta}^{\theta}$ is selfadjoint with positive spectrum.

After introducing $\tau_{\theta}$ for $|\theta|<\pi / 4$ on $D_{0}$, we may define $D_{\theta}$ in $\mathscr{H}_{\theta}$ to be the set of all finite linear combinations of $\Psi_{\theta}(n, m ; \theta)\left(=\Psi_{0}(n, m ; \theta)\right.$ if $\left.|\theta|<\pi / 4\right)$. If $|\varphi|<\pi / 4$, we can introduce a positive definite inner product on $D_{\theta}$ uniquely defined by

$$
\left(\Psi_{\theta}(n, m ; \theta), \Psi_{\theta}\left(n^{\prime}, m^{\prime} ; \theta\right)\right)_{\theta+\varphi}=\left(\Psi_{\theta}(n, m ; \theta-\varphi), \Psi_{\theta}\left(n^{\prime}, m^{\prime} ; \theta-\varphi\right)\right)_{\theta},
$$

and define $\mathscr{H}_{\theta^{\prime}}$ for $\theta^{\prime}=\theta+\varphi$ and $\bar{H}_{\theta^{\prime \prime}}^{\theta^{\prime}}$. As long as $\left|\theta^{\prime}\right|<\pi / 4$, $\mathscr{H}_{\theta^{\prime}}$ and $\bar{H}_{\theta^{\prime \prime}}^{\theta^{\prime}}$ coincide with the earlier definition. However this extends the definition of $\mathscr{H}_{\theta}$ beyond the restriction $|\theta|<\pi / 4$. By continuing this process we can define $\mathscr{H}_{\theta}$ recursively for all $\theta$. The operator $\bar{H}_{\theta}^{\theta}$ is then always positive selfadjoint (with a pure point spectrum at non-negative integers).

Remark 5. If a sequence in $D_{0}$ converges to 0 relative to $\tau_{\theta_{1}}$ and is a Cauchy sequence relative to $\tau_{\theta_{2}}$, then it converges to 0 relative to $\tau_{\theta_{2}}$ (Lemma 6). Thus we can identify a point in $\mathscr{H}_{\theta_{1}}$ and a point in $\mathscr{H}_{\theta_{2}}$ if they are the limit of the same sequence in $D_{0}$ relative to $\tau_{\theta_{1}}$ and $\tau_{\theta_{2}}$, respectively, and we can speak of $\mathscr{H}_{\theta_{1}} \cap \mathscr{H}_{\theta_{2}}\left(\left|\theta_{1}\right|,\left|\theta_{2}\right|<\pi / 4\right)$.

\section{Computations}

Lemma 1. $h \equiv\left(a b-a^{*} b^{*}\right)$ is essentially selfadjoint in $\mathscr{H}_{0}$ and vectors in $D_{0}$ are analytic vectors of $h$.

Proof. $a^{*}, a,-b^{*}, b$ satisfy the ordinary commutation relations for creation and annihilation operators, with $a$ and $b$ annihilating $\Psi_{0}(0,0 ; 0)$. Since $h$ is a symmetric quadratic expression in $a^{*}, a,-b^{*}, b$, it is well known that vectors in $D_{0}$ (i.e. vectors with a finite number of particles) are analytic vectors for $h$ and $h$ is essentially selfadjoint by Nelson's theorem.

Lemma 2. For $|\theta|<\pi / 4, \Psi_{0}(0,0 ; 0)$ is in the domain of $e^{\theta \bar{h}}(\bar{h}$ denotes the closure of $h)$ and

$$
e^{\theta \bar{h}} \Psi_{0}(0,0 ; 0)=\sum_{p=0}^{\infty}(-1)^{p}(p ! \cos \theta)^{-1}(\tan \theta)^{p} \Psi_{0}(p, p ; 0) .
$$

Proof. Let the right hand side be denoted $f(\theta)$. Since $\left\|\Psi_{0}(p, p ; 0)\right\|_{0}=p$ !, the sum is absolutely convergent, and the convergence is uniform over $\theta$ in any compact subset of $\{\theta ;-\pi / 4<\operatorname{Re} \theta<\pi / 4\}$. Hence $f(\theta)$ is analytic for $-\pi / 4<\operatorname{Re} \theta<\pi / 4$. The same type of estimate shows the convergence of the sum even if each term is multiplied by a polynomial of $a, a^{*}, b$ and $b^{*}$. Hence $f(\theta)$ is in the domain of $\bar{h}$ and a simple computation shows $f^{\prime}(\theta)=\bar{h} f(\theta)$. This implies that $\left(e^{-\theta \bar{h}} \Psi, f(\theta)\right)$ is independent of $\theta$ for any entire vector $\Psi$ and hence $\left(e^{-\theta \bar{h}} \Psi, f(\theta)\right)=(\Psi, f(0))$. Hence $f(\theta)$ is in the domain of $e^{-\theta \bar{h}}$ and $e^{-\theta \bar{h}} f(\theta)=f(0)$. Therefore $f(0)=\Psi_{0}(0,0 ; 0)$ is in the domain of $e^{\theta \bar{h}}$ and (3.1) holds. 
Lemma 3. For any polynomial $P=P\left(a, b, a^{*}, b^{*}\right), P \Psi_{0}(0,0 ; 0)$ is an analytic vector of $\bar{h}$ and, for $|\theta|<\pi / 4$,

$$
P\left(\bar{a}_{\theta}, \bar{b}_{\theta}, \bar{a}_{\theta}^{*}, \bar{b}_{\theta}^{*}\right) e^{\theta \bar{h}} \Psi_{0}(0,0 ; 0)=e^{\theta \bar{h}} P\left(a, b, a^{*}, b^{*}\right) \Psi_{0}(0,0 ; 0) .
$$

Proof. Let $F(\theta)$ be the left hand side. As stated in the proof of Lemma 2, $e^{\theta \bar{h}} \Psi_{0}(0,0 ; 0)$ is in the domain of $P\left(\bar{a}_{\theta}, \bar{b}_{\theta}, \bar{a}_{\theta}^{*}, \bar{b}_{\theta}^{*}\right)$ and $F(\theta)$ is holomorphic in $\theta$ for $|\theta|<\pi / 4$. Furthermore,

$$
[A, \bar{h}]=-(d / d \theta) A
$$

for $A=a_{\theta}, a_{\theta}^{*}, b_{\theta}, b_{\theta}^{*}$, which implies

$$
F^{\prime}(\theta)=\bar{h} F(\theta) .
$$

By the same argument as the proof of Lemma 2, $F(0)$ is in the domain of $e^{\theta \bar{h}}$ and $F(\theta)=e^{\theta \vec{h}} F(0)$, i.e. (3.2) holds. Since $F(\theta)$ is holomorphic for $|\theta|<\pi / 4, F(0)$ is an analytic vector of $\bar{h}$.

Proof of Theorem 1 (1). Let $-\pi / 4<\theta<\pi / 4$ and

$$
\Psi_{0}(n, m ; \theta)=e^{\theta \bar{h}} \Psi_{0}(n, m ; 0) .
$$

Due to (3.2) and $\Psi_{0}(n, m ; 0)=\left(a^{*}\right)^{n}\left(b^{*}\right)^{m} \Psi_{0}(0,0 ; 0)$, we obtain

$$
\bar{H}_{0}^{0} \Psi_{0}(n, m ; \theta)=e^{\theta \bar{h}} H_{0} \Psi_{0}(n, m ; 0)=(n+m+1) \Psi_{0}(n, m ; \theta) .
$$

If $\left|\theta^{\prime}\right|<\min (\theta+\pi / 4,(\pi / 4)-\theta)$, then the analyticity of (3.5) for $|\theta|<\pi / 4$ (for complex $\theta)$ implies

$$
\Psi_{0}\left(n, m ; \theta-\theta^{\prime}\right)=\sum_{k=0}^{\infty}(k !)^{-1}\left(-\theta^{\prime} \bar{h}\right)^{k} \Psi_{0}(n, m ; \theta),
$$

where the sum has to converge. By (3.2), we have

$$
\left.\begin{array}{l}
a_{\theta} \Psi_{0}(n, m ; \theta)=n \Psi_{0}(n-1, m ; \theta) \quad(=0 \quad \text { if } \quad n=0), \\
a_{\theta}^{*} \Psi_{0}(n, m ; \theta)=\Psi_{0}(n+1, m ; \theta), \\
b_{\theta} \Psi_{0}(n, m ; \theta)=-m \Psi_{0}(n, m-1 ; \theta)(=0 \quad \text { if } \quad m=0), \\
b_{\theta}^{*} \Psi_{0}(n, m ; \theta)=\Psi_{0}(n, m+1 ; \theta) .
\end{array}\right\}
$$

Since $\bar{h}$ can be expressed as a second degree polynomial in $a_{\theta}, a_{\theta}^{*}, b_{\theta}$ and $b_{\theta}^{*},(3.7)$ shows that $\Psi_{0}\left(n, m ; \theta-\theta^{\prime}\right)$ is in the closure of the linear span of $\Psi_{0}(n, m ; \theta)$, $n=0,1, \ldots, m=0,1, \ldots$ By repeating the argument starting each time with new $\Psi_{0}\left(n, m ; \theta-\theta^{\prime}\right)$, we conclude that $\Psi_{0}(n, m ; 0)$ is in the closure of the linear span of $\Psi_{0}(n, m ; \theta)$ and hence the completeness of $\Psi_{0}(n, m ; \theta)$.

Remark 6. By a standard formal computation, we obtain

$$
e^{\theta h}=e^{-(\tan \theta) a^{*} b^{*}} e^{-(\log \cos \theta) H_{0}} e^{(\tan \theta) a b},
$$

$$
\begin{aligned}
\Psi_{0}(n, m ; \theta)= & \sum_{p=0}^{\infty} \sum_{q=0}^{\min (n, m)}(-1)^{p}[q !(n-q) !(m-q) ! p !]^{-1} \\
& \cdot n ! m !(\tan \theta)^{p+q}(\cos \theta)^{-(n+m-2 q+1)} \Psi_{0}(n-q+p, m-q+p, 0) .
\end{aligned}
$$

We do not use this formula in this paper. 
Proof of Theorem 1 (2). We introduce the following canonical variables (for which $\mathscr{H}_{0}$ is equivalent to the Schrödinger representation).

$$
\begin{aligned}
& x_{1}=2^{-1}\left(a+a^{*}+i\left(b+b^{*}\right)\right), p_{1}=2^{-1}\left(i\left(a^{*}-a\right)-\left(b^{*}-b\right)\right), \\
& x_{2}=2^{-1}\left(a+a^{*}-i\left(b+b^{*}\right)\right), p_{2}=2^{-1}\left(i\left(a^{*}-a\right)+\left(b^{*}-b\right)\right) .
\end{aligned}
$$

Note that the adjoint $b^{+}$relative to $(,)_{0}$ satisfies $b^{\dagger} \supset-b^{*}$. We then have

$$
\begin{gathered}
H_{\pi / 4}=a^{*} b^{*}+a b=-i\left(h_{1}-h_{2}\right), \\
h_{j}=2^{-1}\left(x_{j}^{2}-p_{j}^{2}\right), \quad j=1,2 .
\end{gathered}
$$

It is known that each $\overline{h_{j}}$ is selfadjoint and has an absolutely continuous spectrum on the whole real line.

Lemma 4. For $-\pi / 4<\theta<\pi / 4$, the following holds.

$$
\begin{aligned}
\left\langle\Psi_{0}(n, m ; \theta), \Psi_{0}\left(n^{\prime}, m^{\prime} ; \theta\right)\right\rangle & =n ! m !(-1)^{m} \text { if } n=n^{\prime}, m=m^{\prime} \\
& =0 \text { otherwise. }
\end{aligned}
$$

Proof. Since $\eta_{0} \bar{h}=-\bar{h} \eta_{0},(3.5)$ implies

$$
(d / d \theta)\left\langle\Psi_{0}(n, m ; \theta), \Psi_{0}\left(n^{\prime}, m^{\prime} ; \theta\right)\right\rangle=\left(\Psi_{0}(n, m ; \theta),\left(\bar{h} \eta_{0}+\eta_{0} \bar{h}\right) \Psi_{0}\left(n^{\prime}, m^{\prime} ; \theta\right)\right)_{0}=0 .
$$

Hence

$$
\left\langle\Psi_{0}(n, m ; \theta), \Psi_{0}\left(n^{\prime}, m^{\prime} ; \theta\right)\right\rangle=\left\langle\Psi_{0}(n, m ; 0), \Psi_{0}\left(n^{\prime}, m^{\prime} ; 0\right)\right\rangle .
$$

Lemma 5. The inner product $(,)_{\theta}$ defined by (2.11) is positive definite on $D_{0}$. A linear map $U_{\theta, 0}$ from $\mathscr{H}_{0}$ onto $\mathscr{H}_{\theta}$ satisfying

$$
U_{\theta, 0} \Psi_{0}(n, m ;-\theta)=\Psi_{0}(n, m ; 0)
$$

exists, is unique and is unitary.

Proof. The positive definiteness of $(,)_{0}$ is immediate from the definition. Since $\eta_{0}^{2}=1,\langle$,$\rangle is nondegenerate in \mathscr{H}_{0}$. Lemma 4 shows linear independence of $\Psi_{0}(n, m ;-\theta)$. By $(2.11), U_{\theta}$ is isometric on the linear span of $\Psi_{0}(n, m ;-\theta)$ and its image is the entire linear span of $\Psi_{0}(n, m ; 0)$. The linear independence of $\Psi_{0}(n, m ;-\theta)$ and the positive definiteness of $(,)_{0}$ then imply the positive definiteness of $(,)_{\theta}$ on $D_{0}$. The completeness of $\Psi_{0}(m, n ;-\theta)$ then implies the existence, uniqueness and unitarity of $U_{\theta, 0}$.

Proof of Theorem 2. Let $\left|\theta^{\prime}\right|<\min (\theta+\pi / 4,(\pi / 4)-\theta)$. By (3.7) and the unitarity of $U_{\theta, 0}$, we have the $\tau_{\theta}$-convergence and $\theta^{\prime}$-analyticity of

$$
\begin{aligned}
U_{\theta, 0} \Psi_{0}\left(n, m ;(-\theta)-\left(-\theta^{\prime}\right)\right) & =\sum_{k=0}^{\infty}(k !)^{-1} U_{\theta, 0}\left(\theta^{\prime} \bar{h}\right)^{k} \Psi_{0}(n, m ;-\theta) \\
& =\sum_{k=0}^{\infty}(k !)^{-1}\left(\theta^{\prime} \overline{h_{\theta}}\right)^{k} U_{\theta, 0} \Psi_{0}(n, m ;-\theta) \\
& =\sum_{k=0}^{\infty}(k !)^{-1}\left(\theta^{\prime} \bar{h}\right)^{k} \Psi_{0}(n, m ; 0)=e^{\theta^{\prime} \bar{h}} \Psi_{0}(n, m ; 0),
\end{aligned}
$$


where $h_{\theta}$ denotes $a_{\theta} b_{\theta}-a_{\theta}^{*} b_{\theta}^{*}$ which is the same as $h$. We denote this vector by $\Psi_{\theta}\left(n, m ; \theta^{\prime}\right)$. By the same method as the proof of (3.6), we obtain

$$
\bar{H}_{\theta^{\prime}}^{\theta} \Psi_{\theta}\left(n, m ; \theta^{\prime}\right)=(n+m+1) \Psi_{\theta}\left(n, m ; \theta^{\prime}\right) .
$$

The completeness of $\Psi_{\theta}\left(n, m ; \theta^{\prime}\right)$ in $\mathscr{H}_{\theta}$ follows from the completeness of $\Psi_{0}\left(n, m ; \theta^{\prime}-\theta\right)$ in $\mathscr{H}_{0}$ and the unitarity of $U_{\theta, 0}$.

If $|\theta|<\pi / 4$, then $\Psi_{\theta}(n, m ; \theta)=U_{\theta, 0} \Psi_{0}(n, m ; 0)$ is a complete orthonormal system and $\bar{H}_{\theta}^{\theta}$ is positive selfadjoint.

The system of $\Psi_{\theta}(n, m ; \theta), a_{\theta+\varphi}, a_{\theta+\varphi}^{*}, b_{\theta+\varphi}, b_{\theta+\varphi}^{*}$ and $\bar{H}_{\theta+\varphi}^{\theta}$ in $\mathscr{H}_{\theta}$ is unitarily equivalent to the system of $\Psi_{0}(n, m ; 0), a_{\varphi}, a_{\varphi}^{*}, b_{\varphi}, b_{\varphi}^{*}$ and $\bar{H}_{\varphi}^{0}$ in $\mathscr{H}_{0}$ through $U_{\theta, 0}$. Thus we immediately see that $\bar{H}_{\theta+\varphi}^{\theta}$ has a complete set of eigenvectors $\Psi_{\theta}(n, m ; \theta+\varphi)=U_{\theta, 0} \Psi_{0}(n, m ; \varphi)$ belonging to eigenvalues $n+m+1$. Furthermore in exactly the same manner as before we can construct a unitary map $U_{\psi, \theta}$ through which the system of $\Psi_{\psi}(n, m ; \psi+\varphi), a_{\psi+\varphi}, a_{\psi+\varphi}^{*}, b_{\psi+\varphi}, b_{\psi+\varphi}^{*}$, and $\bar{H}_{\psi+\varphi}^{\psi}$ in $\mathscr{H}_{\psi}$ is unitarily equivalent to the system with $\psi$ changed to $\theta$. Furthermore $U_{\psi, \theta} U_{\theta, 0}=U_{\psi, 0}$ whenever $|\psi|<\pi / 4$. Therefore Theorem 2 follows from Theorem 1.

Lemma 6. The indefinite inner product has a unique continuous extension in $\mathscr{H}_{\theta}$. If $\Phi_{n} \in D_{0}$ converges to 0 in $\tau_{\theta}$ topology and $\Phi=\lim \Phi_{n}\left(\in \mathscr{H}_{\theta^{\prime}}\right)$ in $\tau_{\theta^{\prime}}$ topology, then $\Phi=0$.

Proof. Let $\eta_{\theta} \Psi_{\theta}(n, m ; \theta)=(-1)^{m} \Psi_{\theta}(n, m ; \theta)$ and

$$
\langle\Psi, \Phi\rangle_{\theta}=\left(\Psi, \eta_{\theta} \Phi\right)_{\theta} .
$$

Since $\Psi_{\theta}(n, m ; \theta)$ is a complete orthonormal basis in $\mathscr{H}_{\theta}$ and $\eta_{\theta}^{2}=1$, it defines a nondegenerate $\tau_{\theta}$-continuous indefinite inner product in $\mathscr{H}_{\theta}$. Furthermore, the same proof as Lemma 4 implies that

$$
\begin{aligned}
\left\langle\Psi_{\theta}(n, m ; \theta+\varphi), \Psi_{\theta}\left(n^{\prime}, m^{\prime} ; \theta+\varphi\right)\right\rangle_{\theta} & =\left\langle\Psi_{\theta}(n, m ; \theta), \Psi_{\theta}\left(n^{\prime}, m^{\prime} ; \theta\right)\right\rangle_{\theta} \\
& =\left\langle\Psi_{0}(n, m ; 0), \Psi_{0}\left(n^{\prime}, m^{\prime} ; 0\right)\right\rangle .
\end{aligned}
$$

By setting $\varphi=-\theta$, we see that $\langle\Psi, \Phi\rangle_{\theta}=\langle\Psi, \Phi\rangle$ on $D_{0}$. This proves the first half. As a result, we obtain $\left\langle\Psi, \Phi_{n}\right\rangle \rightarrow 0$ for all $\Psi \in D_{0}$ and hence $\langle\Psi, \Phi\rangle_{\theta}=0$ for all $\Psi \in D_{0}$. This implies $\Phi=0$.

\section{References}

1. Haag, R.: Dan. Mat. Fys. Medd. 29, No. 12 (1955)

2. Ito, K.R.: Publ. RIMS Kyoto Univ. 14, 503 (1978)

3. Kato, T.: Perturbation theory for linear operators. Berlin, Heidelberg, New York: Springer 1966

Communicated by R. Jost

Received November 12, 1981 